\title{
Desempenho Cognitivo na Infância: A Mãe e o Meio Urbano fazem a Diferença ${ }^{1}$ \\ ARTIGO ORIGINAL
}

\author{
Ana Filipa Alves ${ }^{2}$ \\ Gina C. Lemos \\ Universidade do Minho \\ Lurdes Brito \\ Grande Colégio Universal, Porto \\ Ana A. Martins \\ Leandro S. Almeida \\ Universidade do Minho
}

\begin{abstract}
RESUMO - Apesar das controvérsias sobre o conceito e a avaliação da inteligência, o desempenho cognitivo assume um papel fundamental no contexto educativo e são múltiplos os fatores que lhe estão associados. Este estudo toma uma amostra aleatória e representativa de 1201 crianças do $2{ }^{\circ}$ ciclo do ensino básico de escolas públicas portuguesas, com idades entre 9 e 14 anos. Discute-se o impacto das variáveis sociofamiliares (profissão da mãe e do pai, escolaridade da mãe e do pai e meio de pertença urbano vs rural). Os resultados destacam a relevância dessas variáveis para a explicação do desempenho cognitivo dos alunos, especialmente a escolaridade da mãe e o meio urbano de proveniência. Apresentam-se considerações práticas voltadas à equidade do sistema educativo.
\end{abstract}

Palavras-chave: desenvolvimento intelectual, aptidão cognitiva, aprendizagem, fatores socioculturais, nível socioeconômico

\section{Cognitive Performance in Childhood: Mother and Urban Environment Make the Difference}

\begin{abstract}
Despite the controversies over the concept and evaluation of intelligence, cognitive achievement plays a fundamental role in educational context, and multiple factors are related to it. The current study uses a random and representative sample of 1201 2nd grade children from Portuguese public schools, between 9 and 14 years old. This study aims to discuss the impact of social and familial variables (mother and father's jobs, mother and father's educational level, and urban or rural environment of belonging). The results highlight the importance of social and familial variables as an explanation to students' cognitive achievement, with great relevance to mother's educational level and urban environment of belonging. Practical considerations aiming a better equity in educational system are presented.
\end{abstract}

Keywords: intellectual development, cognitive ability, learning, sociocultural factors, socioeconomic status

A inteligência é considerada como uma complexa capacidade para pensar, resolver novos problemas, inferir, compreender, reconhecer e construir estruturas, relações, contextos e significado (Rindermann, 2007). Essa capacidade assume um papel particularmente importante no contexto educativo (Kane \& Brand, 2008; Spinath, Spinath, Harlaar, \& Plomin, 2006; Stelzl, Merz, Ehlers, \& Remer, 1995), nomeadamente na explicação do nível de aprendizagem, na compreensão das dificuldades de aprendizagem e do (in)sucesso escolar (Almeida, Guisande, \& Simões, 2007; Seabra-Santos, 2000; Sternberg, 2012).

Contrariamente à visão atual, ao longo da primeira metade do século XX, o (in)sucesso escolar, conceito considerado estável, era comumente explicado em função da capacidade intelectual (Colom \& Flores-Mendoza, 2007), cujo desenvolvimento seria imune à aprendizagem escolar (Ceci, 1991). Embora a investigação na área aponte para uma correlação positiva entre as habilidades cognitivas dos

1 Vários autores integrados no CIEd e apoiados pela FCT.

2 Endereço para correspondência: Instituto de Educação, Universidade do Minho, Campus de Gualtar, Braga, Portugal. CEP: 4710-057. E-mail: afilipaalves@hotmail.com alunos e o seu desempenho acadêmico (Deary, Strand, Smith, \& Fernandes, 2007; Gottfredson, 2002; Lemos, Almeida, Guisande, \& Primi, 2008; Strenze, 2007), presentemente compreende-se que não é possível estabelecer uma relação linear e causal da inteligência para a aprendizagem e o desempenho escolar (Colom \& Flores-Mendoza, 2007).

$\mathrm{O}$ debate inteligência vs rendimento acadêmico tem sido deveras polêmico (Deary et al., 2007), na medida em que, apesar de as variáveis cognitivas poderem explicar o (in) sucesso escolar, não significa, de todo, que a aprendizagem e o rendimento escolar sejam explicados apenas por fatores pessoais dos alunos, particularmente os associados à capacidade intelectual (Barca, Brenlla, Canosa, \& Enriquez, 1999; Ceci, 1991; Chamorro-Premuzic \& Arteche, 2008). Vários estudos têm demonstrado que o desempenho nos testes é influenciado, por exemplo, por variáveis sociodemográficas (Freitas, Simões, Alves, \& Santana, 2012; Nisbett et al., 2012), nomeadamente a comunidade e a classe social de pertença. Nesse sentido, as funções cognitivas mais clássicas parecem ser manifestamente insuficientes para explicar todo o comportamento inteligente. Os aspetos culturais e a gestão dos conhecimentos sociais influenciam aquilo que podemos 
designar por comportamento adaptativo ou inteligente (Almeida, Guisande, \& Ferreira, 2009; Sternberg, 2012).

Os primeiros estudos científicos referentes à inteligência apresentam uma base eminentemente cognitivista (Almeida et al., 2009). As mais recentes abordagens ecológicas do desenvolvimento psicológico destacam o papel fundamental das variáveis socioculturais na cognição. Ambos os componentes, sociais e cognitivos, são cruciais na cognição e na aprendizagem. À luz da abordagem bioecológica, o desenvolvimento humano traduz, para além das características intrapessoais do indivíduo, a influência dos contextos sociais de pertença (Bairrão, 1994; Bronfenbrenner, 1979; Veiga, Galvão, Festas, \& Taveira, 2012). Nesse quadro, e sobretudo durante a infância, a família como contexto social primário exerce um papel preponderante no desenvolvimento da criança e, naturalmente, no seu desenvolvimento cognitivo e na aprendizagem (Andrade et al., 2005; Cruz et al., 2011; Phillipson, 2009, 2010; Pomerantz \& Dong, 2006).

Bronfenbrenner (1979), no seu modelo atualmente denominado de bioecológico, enfatiza o estudo da criança no contexto dos ambientes múltiplos nos quais ela se desenvolve, identificando cinco níveis interligados de influência ambiental ou contextos de desenvolvimento, do mais próximo ao mais afastado ou genérico: (a) microssistema - ambiente quotidiano imediato da família, escola ou vizinhança, que inclui relações face a face com pais, irmãos, amigos, professores, em que a influência é interativa; (b) mesossistema - interação de vários microssistemas em que a criança está inserida. Pode incluir relações entre casa e escola, como reuniões de pais e professores ou relações entre família e o grupo de pares; (c) exossistema - ligações entre dois ou mais contextos em que, pelo menos em um, a criança não está inserida, mas a afeta indiretamente, como emprego dos pais; (d) macrossistema-padrões culturais, tais como crenças, ideologias e sistemas económicos e políticos; (e) cronossistema - engloba a dimensão tempo, enquadrando todas as dinâmicas dos vários sistemas ou ambientes em que a criança se move e desenvolve, ou seja, influência da mudança ou estabilidade, normativa ou não normativa, na criança e no meio. Pode incluir alterações na estrutura familiar, local de residência, emprego dos pais, assim como mudanças culturais ou económicas (Collins Maccoby, Steinberg, Heatherington, $\&$ Bornstein, 2000). De acrescentar que todos esses sistemas continuam a ser entendidos como reciprocamente interativos (Bairrão, 1994; Harris, 1995; Vandell, 2000; Tudge, Mokrova, Hatfield, \& Karnik, 2009). Com efeito, o contexto familiar e o contexto escolar, enquanto sistemas imediatos da criança, interagem reciprocamente e assumem um papel primordial no desenvolvimento e na aprendizagem (Bronfenbrenner \& Morris, 2006), sobretudo durante os anos pré-escolares e escolares (Johnson, 2010).

\section{Relevância das Variáveis Familiares}

Embora, nas últimas décadas, a família tenha sofrido grandes modificações e apareça algumas vezes diminuída na sua relevância, esta inquestionavelmente constitui um espaço educativo por excelência (Bairrão, 1994), com um papel significativo no desenvolvimento cognitivo da criança. Trata-se de espaço privilegiado para a elaboração e aprendizagem de dimensões significativas da interação intencional e cognitiva, como, por exemplo, a linguagem, a comunicação, as relações interpessoais, as normas sociais e a aprendizagem escolar (González, 2011; Talib, Mohamad, \& Mamat, 2011). Na verdade, vários estudos demonstram que os padrões culturais, a linguagem, o processo de socialização da criança no seio da família e da comunidade de pertença, o grau de aproximação das práticas educativas familiares e dos padrões exigidos e estimulados pela escola, são fortes condicionantes da aprendizagem e do rendimento escolar dos alunos (Freijo et al., 2008; Hoover-Dempsey \& Sandler, 1995).

Sob a designação de dimensões sociais, podemos facilmente associar hábitos e estilos de vida no seio familiar, linguagem e atitudes face ao conhecimento e à escola, condições de vida e acesso a bens culturais e educativos, entre outros, à qualidade da aprendizagem e ao sucesso escolar das crianças (Cheung \& Andersen, 2003; De Graaf, De Graaf, \& Kraaykamp, 2000; Hoover-Dempsey \& Sandler, 1995; Jaeger, 2009; Myrberg \& Rosén, 2009; Roscigno \& Ainsworth-Darnell, 1999). Nesse âmbito, o insucesso escolar é, muitas vezes, explicado pela ausência de capital cultural das famílias de onde são oriundos os alunos, estando o sucesso escolar mais garantido quando, na família, encontram-se percepções, orientações, disposições, valores e hábitos culturais viabilizados pela ou na interação com a escola (Bairrão, 1994; Lareau \& Horvat, 1999; Sullivan, 2001). Os hábitos de vida, os modelos de interação familiar, as expetativas educativas em relação à aprendizagem dos filhos, a natureza e a extensão do envolvimento parental na educação e na aprendizagem dos filhos assim como a qualidade do apoio nas tarefas escolares influenciam o desenvolvimento cognitivo e psicossocial e constituem-se como fatores importantes na diferenciação de padrões de realização cognitiva e acadêmica (e.g. Davis-Kean, 2005; Galindo \& Sheldon, 2012; Guttman \& McLoyd, 2000; Jeynes, 2005; Rimkute, Hirvonen, Tolvanen, Aunola, \& Nurmi, 2012; Rothon, Arephin, Klineberg, Cattell, \& Stansfeld, 2011; Stull, 2013; Zhan, 2006).

De uma forma mais direta, a família promove a aprendizagem através do estímulo à expressão oral e escrita, incentivando a leitura, promovendo a conversação, a exploração e a discussão de ideias. No entanto, também o faz, de forma mais indireta, através da valorização implícita e explícita da aprendizagem acadêmica e da vida escolar em geral ou da associação do sucesso acadêmico à realização e satisfação profissionais (Chiu \& Xihua, 2008; De Graaf et al., 2000; Jadue, 2002; Myrberg \& Rosén, 2009; Roscigno \& Ainsworth-Darnell, 1999; Santos \& Graminha, 2005).

Estudos das últimas décadas do século passado apontavam já para as variáveis familiares, nomeadamente a escolaridade dos pais e o rendimento familiar, como particularmente associadas ao desempenho cognitivo (Lordelo, Chalhub, Guirra, \& Carvalho, 2007; Mercy \& Steelman, 1982; Scarr \& Weinberg, 1978; Sellers, Burns, \& Guyrke, 1996; Vanderploeg, Schinka, Baum, Tremont, \& Mittenberg. 1998). Se, em alguns estudos, o melhor preditor da realização cognitiva se refere à escolaridade da mãe (Gutman, Sameroff, \& Cole, 2003; Lordelo et al., 2007; Lordelo, Fonseca, \& 
Araújo, 2000; Mercy \& Steelman, 1982; Ribas, Moura, \& Bornstein, 2003), em outros, a escolaridade da mãe e a escolaridade do pai contribuíam de igual forma para a explicação dos resultados cognitivos (Scarr \& Weinberg, 1978; Sellers et al., 1996; Vanderploeg et al., 1998).

No que concerne ao impacto da escolaridade da família na realização intelectual dos filhos, o racional subjacente é que ter pais com maior escolaridade parece aumentar a probabilidade de a criança contar com um ambiente mais estimulante, o que se repercute no seu desenvolvimento cognitivo e, consequentemente, na aprendizagem (Cianci, Orsini, Hulbert, \& Pezzuti, 2013; Rindermann, Michou, \& Thompson, 2011; Umek, Podlesek, \& Fekonja, 2005). Com efeito, estudos mostram que, comparativamente a pais com baixa escolaridade, os pais com escolaridade mais elevada proporcionam ambientes e experiências cognitivamente mais estimulantes (Bradley \& Corwyn, 2000; Bradley, Corwyn, Burchinal, McAdoo, \& Coll, 2001), investem mais na vida escolar dos seus filhos (Davis-Kean, 2005; Dumka, Gonzales, Bonds, \& Millsap, 2010; Engin-Demir, 2009), facilitam deliberadamente o desenvolvimento cognitivo e a autonomia das crianças, favorecem a sua autoestima, incentivam o esforço e os métodos de estudo, ajudando a fixar metas escolares, a apresentar atribuições causais apropriadas na explicação dos resultados acadêmicos e a desenvolver uma motivação intrínseca (Chiu \& Xihua, 2008; García \& Sánchez, 2005; Stull, 2013).

As condições socioeconômicas da família apresentam uma poderosa influência no desenvolvimento cognitivo da criança (Bradley \& Corwyn, 2002; Burger, 2010; Cabrera, Shannon, \& Tamis-LeMonda, 2007; Conselho Nacional de Educação [CNE], 2013; Strenze, 2007). No limite, as crianças expostas a condições sociofamiliares desfavorecidas têm uma maior probabilidade de não adquirir as competências necessárias para posterior sucesso educativo (Burchinal, Roberts, Hooper, \& Zeisel, 2000; Gutman et al., 2003). Os investigadores sugerem que crianças provenientes de níveis socioeconômicos baixos têm um acesso quase nulo a materiais, experiências e contextos cognitivamente estimulantes, o que constrange o seu desenvolvimento cognitivo (Jimerson, Egeland, Sroufe, \& Carlson, 2000; Santos \& Graminha, 2005). Dados do National Longitudinal Survey of Youth e do National Household Education Survey (Bradley et al., 2001) indicam que as crianças provenientes de famílias desfavorecidas têm menos acesso a uma variedade de materiais lúdicos e pedagógicos, desde a infância até à adolescência. Para além disso, a probabilidade de viajarem, visitarem museus ou bibliotecas, ou participarem em eventos educativos e culturais parece ser francamente menor. De fato, a literatura aponta para o potencial catalisador do acesso a esse tipo de materiais/recursos culturais na relação que se estabelece entre o nível socioeconômico e a realização cognitiva e acadêmica da infância à adolescência (Guo \& Harris, 2000; Lemos, Almeida, \& Colom, 2011).

\section{Relevância das Variáveis Sociais}

Os padrões de vida, as oportunidades educativas e as experiências culturais ganham um cunho característico em função do meio em que os indivíduos se inserem e daí decorrem, naturalmente, práticas educativas e expetativas sociais distintas (Bairrão, 1994; Hoover-Dempsey \& Sandler, 1995). A literatura é relativamente consensual ao sugerir diferenças cognitivas dos indivíduos de acordo com as categorias sociais de pertença, incluindo o grupo étnico, o tipo de comunidade, a classe social e a escolaridade, embora permaneçam algumas controvérsias (Deary et al., 2005; Strenze, 2007; Veiga et al., 2012).

Os estudos até então realizados que procuram relacionar a influência do meio sociocultural e o desempenho cognitivo das crianças apontam para melhores resultados nos testes de inteligência em indivíduos pertencentes ao meio urbano, com níveis de escolarização médios mais elevados e mais favorecidos economicamente, de forma que o (in)sucesso escolar não atinge de igual forma todas as classes e grupos sociais (Lemos, 2007; Strenze, 2007; Weschler, 2003). Alunos de classes sociais mais desfavorecidas tendem a apresentar uma atitude negativa em relação à escola, com baixas aspirações e poucas expetativas, menor motivação e maior dificuldade na realização das tarefas escolares bem como inadequada socialização familiar, muitas vezes por serem pouco estimulados para a intelectualização dos seus atos e para o desenvolvimento da linguagem (GarciaBacete, 2003). Os alunos provenientes de meio sociocultural desfavorecido, muitas vezes, enfrentam ambientes familiares pouco estruturados e com problemas sociais graves. Presumivelmente, nesse ambiente de risco, onde as restrições de oportunidades e estimulação se destacam, não usufruirão de uma base cultural necessária para triunfar na escola (Blin \& Gallais, 2005), acabando esta por ser acusada de algum efeito seletivo e reprodutivo das diferenças sociais. De fato, frequentemente a escola apresenta um papel seletivo, não de acordo com as capacidades dos alunos, mas pelo grupo social de pertença, contribuindo desse modo para a reprodução da estrutura social (Sullivan, 2001).

As diferenças nos níveis de desempenho cognitivo em função do caráter urbano versus rural tendem a não ser encaradas como déficits intelectuais. A literatura pondera diversos condicionalismos que poderão cooperar para a explicação de tais discrepâncias, entre os quais se salientam a verbalidade ou não verbalidade dos itens utilizados na avaliação, o grau de adequação do comportamento dos indivíduos à situação de teste e o grau de acessibilidade a "oportunidades ambientais", promotoras do desenvolvimento cognitivo (no seio da família e/ou em contextos mais amplos de educação e socialização; Lemos, 2007; O’Connor \& McCartney, 2007). De maneira geral, esse viés nos resultados parece decorrer do formato e do conteúdo dos itens utilizados, sobretudo quando verbais ou associados ao desenvolvimento da linguagem, sendo que as crianças dos meios urbanos ou mais favorecidos tendem a envolver-se de forma mais genuína e espontânea na resolução dessas tarefas (Lemos, 2007; Roazzi \& Souza, 2002; Te Nijenhuis, Evers, \& Mur, 2000).

Sintetizando, crianças pertencentes a ambiente familiares e a contextos sociais mais favorecidos, econômica e culturalmente, beneficiam de mais oportunidades de interação com as ferramentas e os artefatos de uma cultura dominante, podendo, dessa forma, otimizar o seu desenvolvimento 
cognitivo e a sua realização escolar (Blin \& Gallais, 2005; Guo \& Harris, 2000; Lemos et al., 2011). De forma a superar as dificuldades que crianças menos favorecidas geralmente apresentam, "a problemática da educação dos 0 aos 12 anos deverá ser encarada sob o duplo ângulo da melhoria da sua qualidade e da garantia da equidade do sistema" (CNE, 2009, p. 20).

Este estudo pretende contribuir para a discussão sobre o impacto de variáveis familiares e sociais no desenvolvimento cognitivo em uma faixa etária que não tem sido sistematicamente pesquisada, correspondente à transição da infância para a adolescência. Assim, o presente estudo propõe apreciar a importância relativa de variáveis sociofamiliares (profissão da mãe, profissão do pai, habilitação escolar da mãe, habilitação escolar do pai, e caráter urbano ou rural da comunidade de pertença) no desempenho cognitivo de um grupo aleatório e representativo de alunos portugueses, com idades compreendidas entre 9 e 14 anos.

\section{Método}

\section{Participantes}

Participaram, neste estudo, 1201 crianças do 2. ${ }^{\circ}$ Ciclo do Ensino Básico (CEB), com idades compreendidas entre os 9 e os 14 anos $(M=11,0 ; D P=0,98)$, provenientes de escolas públicas de Portugal Continental. A constituição da amostra cumpriu com os critérios de aleatoriedade e representatividade (quota de referência de 6\%), tomando a distribuição de alunos por zonas do país (Norte, Centro, Lisboa e Vale do Tejo e Sul), meio de proveniência dominante dos alunos (urbano/rural) e ano de escolaridade (5. ${ }^{\circ}$ e $6 .^{\circ}$ anos).

\section{Instrumentos}

Para a avaliação da inteligência, recorreu-se à Bateria de Provas de Raciocínio, na sua versão para o 2. ${ }^{\circ} \mathrm{CEB}$ (BPR5/6; Almeida \& Lemos, 2007). A BPR5/6 surge no sentido de avaliar a realização cognitiva dos alunos que frequentam o $5 .^{\circ}$ e o $6 .^{\circ}$ ano de escolaridade no que se refere à apreensão de relações entre elementos (raciocínio indutivo) e aplicação das relações inferidas a novas situações (raciocínio dedutivo). Essa bateria é formada por quatro provas, que têm em comum a avaliação do raciocínio como operação ou função cognitiva dominante, sendo que diferem no conteúdo ou material em que os itens são formulados. A prova de raciocínio abstrato (RA) é formada por analogias envolvendo figuras geométricas ou traçados gráficos sem qualquer significado aparente (20 itens, $5 \mathrm{~min}$.), que o aluno deve completar ao escolher uma das quatro opções de resposta apresentadas. A prova de raciocínio numérico (RN) é formada por sequências numéricas, lineares ou alternadas (15 itens, $10 \mathrm{~min}$.), que o aluno deve completar. A prova de raciocínio verbal (RV) é formada por analogias tomando um conjunto heterogêneo de relações que se podem estabelecer entre palavras (20 itens, 4 min.), que o aluno deve completar ao escolher uma das quatro alternativas de resposta que o item apresenta. A prova de raciocínio prático ou de resolução de problemas (RP) é constituída por problemas práticos ou do quotidiano com alguma complexidade informativa, que o aluno deve processar para elaborar a sua resposta (15 itens, $10 \mathrm{~min}$.).

No sentido de avaliar o grau em que os resultados da bateria se encontram libertos de erros de medida e sua possibilidade de generalização, procedeu-se à análise da precisão dos resultados, tomando uma subamostra de alunos $(n=115)$ da amostra de aferição nacional através do método da bipartição dos itens, aplicando-se a fórmula corretiva de Spearman-Brown. Os coeficientes de precisão nas provas tendem a situar-se acima de 0,71 , sendo que o índice mais reduzido regista-se na prova $\mathrm{RA}$ e o mais elevado, na prova $\mathrm{RN}(\mathrm{RA}=0,71 ; \mathrm{RN}=0,84 ; \mathrm{RV}=0,72 ; \mathrm{RP}=0,75)$. $\mathrm{Da}$ análise fatorial exploratória é extraído um único fator que explica $60,4 \%$ da variância dos resultados. A análise fatorial confirmatória reforça a unidimensionalidade da bateria: o fator geral de inteligência $(\mathrm{g})$ prediz as quatro provas da BPR5/6 e o modelo ajusta-se perfeitamente.

O levantamento de dados sociofamiliares foi realizado através de itens a serem preenchidos pelo aluno, tais como profissão da mãe, profissão do pai, escolaridade da mãe e escolaridade do pai. Adicionalmente considerou-se a caracterização do meio de proveniência dos alunos por referência ao caráter urbano ou rural da comunidade em que a escola está inserida, de acordo com os dados do Ministério da Educação.

\section{Procedimento}

Inicialmente foi realizado um pedido de autorização às escolas e aos encarregados de educação, acompanhado da explicação da natureza e objetivos de trabalho. Assegurouse o anonimato e a confidencialidade dos dados levantados, bem como o caráter voluntário da participação. Aos alunos também foram explicados os objetivos do estudo, as tarefas a realizar e a importância da sua participação em uma investigação deste âmbito. A bateria foi aplicada no território nacional por uma equipe de psicólogos que receberam formação específica para esse fim. Não surgiram dificuldades na aplicação coletiva das provas, que integram exemplos esclarecedores. Todas as instruções que constam do manual foram rigorosamente seguidas.

Os alunos responderam a um breve questionário para levantamento de dados sociofamiliares, nomeadamente profissão da mãe/pai e escolaridade da mãe/pai. No que toca à profissão do pai e da mãe, consideramos três níveis correspondentes ao nível socioeconômico - baixo, médio e alto -, sendo que, no caso das mães com a atividade profissional "doméstica em casa própria", o procedimento adotado foi fazer coincidir aquele nível profissional com o da atividade profissional do pai do aluno. Por sua vez, no que toca à escolaridade do pai e da mãe, consideramos quatro grupos ${ }^{1}$ : escolaridade até ao $6 .^{\circ}$ ano $\left(2{ }^{\circ} \mathrm{CEB}\right)$, escolaridade

1 Em Portugal, a escolaridade obrigatória é organizada em três níveis do Ensino Básico $\left(1 .^{\circ}, 2 .^{\circ}\right.$ e $3 .^{\circ}$ ciclos, com nove graus) e um nível 
até ao $9 .^{\circ}$ ano $\left(3 .^{\circ} \mathrm{CEB}\right)$, escolaridade até ao $12 .^{\circ}$ ano (Ensino Secundário), e escolaridade superior ao $12 .^{\circ}$ ano (Ensino Superior). A caracterização das escolas por referência à comunidade de pertença tomou dois grupos - caráter urbano e caráter rural - segundo a categorização indicada pelo Ministério da Educação.

de Ensino Secundário (com três graus). Após o Ensino Secundário os alunos podem ingressar no Ensino Superior.

Tabela 1. Resultados nas quatro provas da BPR $5 / 6$ pelos alunos do $5 .^{\circ}$ e $6 .^{\circ}$ anos de escolaridade

\begin{tabular}{lllllll}
\hline & \multicolumn{3}{c}{$5 .^{\circ}$ ano $(\mathrm{N}=616)$} & \multicolumn{3}{c}{$6 .^{\circ}$ ano $(\mathrm{N}=594)$} \\
\cline { 2 - 7 } Provas & $M$ & $D P$ & $\begin{array}{l}\text { Mín.- } \\
\text { Máx. }\end{array}$ & $M$ & $D P$ & $\begin{array}{l}\text { Mín.- } \\
\text { Máx. }\end{array}$ \\
\hline RA & 10,15 & 3,42 & $1-19$ & 11,06 & 3,32 & $1-19$ \\
RN & 6,27 & 2,84 & $1-14$ & 7,28 & 3,05 & $1-15$ \\
RV & 11,61 & 3,34 & $3-20$ & 13,04 & 3,28 & $3-20$ \\
RP & 8,67 & 2,66 & $1-14$ & 9,28 & 2,60 & $1-15$ \\
\hline
\end{tabular}

\section{Resultados}

A tabela 1 apresenta os resultados obtidos (média, desviopadrão, mínimo e máximo) em cada uma das quatro provas da bateria para os dois anos de escolaridade considerados.

Como seria esperado, verificamos que o nível médio de desempenho cognitivo aumenta na transição do $5 .^{\circ}$ para o 6. ${ }^{\circ}$ ano; o que foi constatado em todas as provas da bateria.

No sentido de apreciarmos a contribuição das variáveis sociofamiliares (profissão do pai/mãe, escolaridade do pai/ mãe, meio urbano/rural) no desempenho global dos alunos na BPR5/6, procedemos a uma análise de regressão linear, tomando as variáveis separadamente (método enter). Para considerarmos, em simultâneo, os resultados dos alunos do $5 .^{\circ}$ e do $6 .^{\circ}$ anos, procedemos ao cálculo de "notas z" para a pontuação final da bateria. A tabela 2 sintetiza os resultados da análise de regressão das variáveis sociofamiliares avaliadas que explicam a variância do desempenho cognitivo dos alunos do nível de escolaridade em estudo (2. $\left.{ }^{\circ} \mathrm{CEB}\right)$.
Acrescenta-se que o modelo se apresenta estatisticamente significativo $(F(5,888)=48.69 ; p<0,001)$, sendo que o conjunto de variáveis preditoras explica $22 \%$ da variância dos resultados na pontuação global da bateria.

Conforme se pode observar, os resultados sugerem uma assinalável relevância das variáveis sociofamiliares para a explicação da variância do desempenho cognitivo, mais concretamente em cerca de $22 \%$. Atividades profissionais correspondentes a um nível socioeconômico mais elevado e maiores índices de escolaridade dos pais estão associados a desempenhos cognitivos superiores dos filhos nas provas de raciocínio. Em uma análise mais detalhada das variáveis consideradas no modelo, a profissão do pai não assume significado estatístico no impacto que detém na explicação da realização cognitiva do aluno, ao passo que a profissão da mãe já concorre para explicar a variância dos resultados nas provas cognitivas (com um $p=0,05$ ). Por outro lado, comparativamente, as escolaridades do pai e da mãe apresentam-se como mais decisivas para o desempenho cognitivo dos filhos do que as suas atividades profissionais. À semelhança do que sucedia para a profissão, também a escolaridade da mãe parece assumir um impacto superior à do pai. Finalmente, o meio em que se situa a escola, e tendencialmente a comunidade de pertença da criança, assume também um impacto significativo no seu desempenho cognitivo: o meio urbano está associado a melhores desempenhos nas provas de raciocínio quando comparado com o meio rural.

\section{Discussão e Conclusões}

Neste estudo, foi tomada uma amostra aleatória e representativa de alunos portugueses, na transição da infância para a adolescência. $\mathrm{O}$ seu desempenho cognitivo foi avaliado através de uma bateria composta por quatro provas de raciocínio (abstrato, numérico, verbal e prático; Almeida \& Lemos, 2007). Os dados sociofamiliares e contextuais considerados referem-se à profissão da mãe, profissão do pai, escolaridade da mãe, escolaridade do pai e caráter urbano ou rural da comunidade de pertença. Conforme mencionado previamente, as profissões da mãe e do pai foram classificadas em três categorias correspondentes a três níveis socioeconômicos (baixo, médio e alto), sendo que as escolaridades da mãe e do pai foram classificadas

Tabela 2. Sintese de resultados da análise de regressão linear junto dos alunos do 2. ${ }^{\circ}$ $C E B$

\begin{tabular}{lcccccc}
\hline \multirow{2}{*}{ Preditores } & \multicolumn{2}{c}{$\begin{array}{c}\text { Coeficientes não } \\
\text { estandardizados }\end{array}$} & $\begin{array}{c}\text { Coeficientes } \\
\text { estandardizados }\end{array}$ & t & Signif. \\
\cline { 2 - 4 } & $\mathrm{B}$ & Erro padrão & Beta & & \\
\hline (Constante) & $-0,747$ & 0,104 & & $-7,179$ & 0,000 \\
Profissão pai & 0,056 & 0,044 & 0,048 & 1,261 & 0,208 \\
Profissão mãe & 0,096 & 0,048 & 0,071 & 1,990 & 0,047 \\
Escolaridade pai & 0,145 & 0,042 & 0,162 & 3,494 & 0,001 \\
Escolaridade mãe & 0,210 & 0,039 & 0,245 & 5,432 & 0,000 \\
Meio escolar & $-0,011$ & 0,003 & $-0,116$ & $-3,873$ & 0,000 \\
\hline
\end{tabular}


em quatro grupos $\left(2 .{ }^{\circ} \mathrm{CEB}, 3{ }^{\circ} \mathrm{CEB}\right.$, Ensino Secundário e Ensino Superior). Os resultados obtidos têm vários pontos de interesse.

Em primeiro lugar, os resultados do modelo testado apontam para as variáveis sociofamiliares estudadas como uma potencial e significativa fonte de variação dos desempenhos cognitivos dos alunos. Isso quer dizer que o background acadêmico e a atividade profissional exercida pelos pais do aluno assim como a sua comunidade de pertença (urbana ou rural) têm um impacto significativo no desempenho intelectual do aluno e, ainda, explicam aproximadamente $25 \%$ da variância dos resultados cognitivos. Tais resultados vêm corroborar a literatura que aponta as variáveis sociofamiliares como assumindo relevância fundamental e efeitos diretos e indiretos no desenvolvimento cognitivo, na aprendizagem e no (in)sucesso escolar dos jovens (e.g. Chiu \& Xihua, 2008; CNE, 2013, Galindo \& Sheldon, 2012; Lemos, 2007; Myrberg \& Rosén, 2009; Rindermann et al., 2011; Strenze, 2007; Stull, 2013).

Em segundo lugar, comparativamente, a escolaridade dos pais concorre de forma mais determinante para a realização cognitiva do jovem do que as atividades profissionais que exercem. Em certa medida, esse resultado pode sugerir que pais com níveis de escolarização mais elevados serão mais capazes de proporcionar condições ambientais familiares que favoreçam o desenvolvimento intelectual dos seus filhos do que pais com menores níveis de escolarização ou que tais condições cognitivamente mais estimulantes sejam mais prováveis em famílias com escolaridades mais elevadas (Ceci \& Williams, 1997; Lemos et al., 2011; Rindermann et al., 2011). Ademais, de acordo com diferentes estudos, pais com níveis de escolaridade mais elevados tendem a ter expetativas superiores em relação à realização acadêmica dos seus filhos, promovem, desde cedo no desenvolvimento, valores e hábitos culturais que favorecem o sucesso educativo, envolvem-se mais ativamente, discutem e apoiam os filhos nas suas tarefas escolares diárias; em suma, investem mais na vida escolar dos seus filhos, certos de que essa atitude terá os seus frutos quer no rendimento escolar quer na realização e satisfação profissionais futuras (Chiu \& Xihua, 2008; Davis-Kean, 2005; De Graaf et al., 2000; Lareau \& Horvat, 1999; Rimkute et al., 2012; Rothon et al., 2011; Sullivan, 2001; Zhan, 2006).

Em terceiro lugar, tais fatores sociofamiliares (escolaridade e profissão do pai e da mãe) predizem a realização cognitiva do aluno de forma diferencial em função da figura parental considerada. Seja a escolaridade ou a atividade profissional exercida, são as da mãe que assumem lugar de destaque, como também vem sendo sublinhado pela literatura (Gutman et al., 2003; Lordelo et al., 2007; Lordelo et al., 2000; Ribas et al., 2003).

Além disso, o meio em que a escola se situa revela-se preponderante no desempenho cognitivo dos alunos. Tradicionalmente associado a uma diversidade e disponibilidade de recursos socioculturais mais preponderantes, o meio urbano (comparativamente ao meio rural) tende a ser mais favorável à realização cognitiva dos alunos, indo ao encontro de resultados de outros estudos (Deary et al., 2005; Guo \& Harris, 2000; Lemos, 2007; O’Connor \& McCartney, 2007; Veiga et al., 2012; Strenze, 2007).
Com esses resultados, sendo certo que os desafios atuais da sociedade global, e também a portuguesa, tendem a colocar o ônus nos constrangimentos econômicos e financeiros que atravessam, a verdade é que, para uma maior equidade na educação das nossas crianças, torna-se imperativo compensar os hiatos daquelas que vivem em condições sociofamiliares mais desfavorecidas. Para esse fim, importa identificar precocemente os fatores de risco e os fatores promotores do desenvolvimento cognitivo e da aprendizagem da criança nos múltiplos contextos em que se move; desenvolver programas e ações junto a pais e mães/encarregados de educação sobre a importância dos fatores cognitivos e não estritamente cognitivos no desempenho intelectual e rendimento escolar do jovem (p.ex. reforço de atitudes positivas face ao conhecimento e à escola, apoio nas tarefas escolares, apoio à promoção de hábitos de estudo, reforço de interações parentais que favoreçam a aprendizagem); integrar na formação inicial e contínua de educadores e professores conteúdos e treino de dinâmicas de comunicação e interação capazes de compensar ou viabilizar o capital cultural das famílias dos alunos; promover ações e iniciativas de colaboração entre as escolas, famílias, autarquias e outras instituições da comunidade que apoiem e capitalizem valores e hábitos culturais.

\section{Referências}

Almeida, L. S., \& Lemos, G. C. (2007). Bateria de Provas de Raciocínio. Braga: Universidade do Minho.

Almeida, L. S., Guisande, M. A., \& Ferreira, A. I. (2009). Inteligência: Perspectivas teóricas. Coimbra: Edições Almedina.

Almeida, L., Guisande, M., \& Simões, M. (2007). Validade preditiva dos testes de inteligência: Estudos com a Bateria de Provas de Raciocínio. Psychologica, 45, 71-85.

Andrade, S. A., Santos, D. N., Bastos, A. C., Pedromônico, M. R., Almeida-Filho, N., \& Barreto, M. L. (2005). Ambiente familiar e desenvolvimento cognitivo infantil: Uma abordagem epidemiológica. Revista Saúde Pública, 39(4), 606-611. http:// dx.doi.org/10.1590/S0034-89102005000400014

Bairrão, J. (1994). A perspectiva ecológica na avaliação de crianças com necessidades educativas especiais e suas famílias: $\mathrm{O}$ caso da intervenção precoce. Inovação, 7, 37-48.

Barca, A., Brenlla, J. C., Canosa, S. S., \& Enriquez, A. G. (1999). Estrategias e enfoques de aprendizaje, contextos familiares y rendimiento académico en el alumnado de educación secundaria: Indicadores para un análisis causal. Revista Galego-Portuguesa de Psicoloxía e Educación, 4(3), 229-269. ISSN 1138-1663

Blin, J., \& Gallais-Deulofeu, C. (2005). Classes dificeis. Porto Alegre: Artmed Editora.

Bradley, R. H., \& Corwyn, R. F. (2002). Socioeconomic status and child development. Annual Review of Psychology, 53, 371-399. http://dx.doi.org/10.1146/annurev.psych.53.100901.135233

Bradley, R. H., Corwyn, R. F., Burchinal, M., McAdoo, H. P., \& Coll, C. G. (2001). The home environments of children in the United States. Relations with behavioral development through age 13. Child Development, 72(6), 1868-1886. http://dx.doi. org/10.1111/1467-8624.t01-1-00383 
Brito, L., Almeida, L. S:, Ferreira, A. I., Guisande, M. A. (2011). Contribución de los procesos y contenidos a la diferenciación cognitiva en la infância: Un estudio com escolares portugueses. Infancia y Aprendizaje, 34(3), 323-336. http://dx.doi. org/10.1174/021037011797238540

Bronfenbrenner, U. (1979). The ecology of human development: Experiments by nature and design. Cambridge: Harvard University Press.

Bronfenbrenner, U., \& Morris, P. (2006). The bioecological model of human development. In W. Damon \& R. M. Lerner (Eds.), Handbook of child psychology: Vol. 1. Theoretical models of human development (pp. 795-828). Hoboken, NJ: Wiley.

Burchinal, M. R., Roberts, J. E., Hooper, S., \& Zeisel, S. A. (2000). Cumulative risk and early cognitive development: A comparison of statistical risk models. Development Psychology, 36(6), 793-807. http://dx.doi.org/10.1037/0012-1649.36.6.793

Burger, K. (2010). How does early childhood care and education affect cognitive development? An international review of the effects of early interventions for children from different social backgrounds. Switzerland: University of Fribourg.

Cabrera, N., Shannon, J., \& Tamis-LeMonda, C. (2007). Fathers' influence on their children's cognitive and emotional development: From toddlers to pre-k. Applied Developmental Science, 11(4), 208-213. http://dx.doi. org/10.1080/10888690701762100

Ceci, S. J. (1991). How much does schooling influence general intelligence and its cognitive components? A reassessment of the evidence. Developmental Psychology, 27(5), 703-722. http://dx.doi.org/10.1037//0012-1649.27.5.703

Chamorro-Premuzic, T., \& Arteche, A. (2008). Intellectual competence and academic performance: Preliminary validation of a model. Intelligence, 36(6), 564-573. http://dx.doi. org/10.1016/j.intell.2008.01.001

Cheung, S. Y., \& Andersen, R. (2003). Time to read: Family resources and educational outcomes in Britain. Journal of Comparative Family Studies, 34, 413-433. ISSN: $\quad 0047-$ 2328

Chiu, M. M., \& Xihua, Z. (2008). Family and motivation effects on mathematics achievement: Analyses of students in 41 countries. Learning and Instruction, 18(4), 321-336. http:// dx.doi.org/10.1016/j.learninstruc.2007.06.003

Cianci, L., Orsini, A., Hulbert, S., \& Pezzuti, L. (2013). The influence of parents' education in the Italian standardization sample of the WISC-III. Learning and Individual Differences, 28, 47-53. http://dx.doi.org/10.1016/j.lindif.2013.09.009

Collins, W. A., Maccoby, E. E., Steinberg, L., Heatherington, E. M., \& Bornstein, M. H. (2000). Contemporary research on parenting: The case for nature and nurture. American Psychologist, 55(2), 218-232. http://dx.doi.org/10.1037/0003066X.55.2.218

Colom, R., \& Flores-Mendoza, C. E. (2007). Intelligence predicts scholastic achievement irrespective of SES factors: Evidence from Brazil. Intelligence, 35(3), 243-251. http://dx.doi. org/10.1016/j.intell.2006.07.008

Conselho Nacional de Educação. (2009). A educação das crianças dos 0 aos 12 anos. Lisboa: Editorial do Ministério da Educação.

Conselho Nacional de Educação. (2013). Estado da educação 2012. Autonomia e descentralização. Lisboa: Editorial do Ministério da Educação e Ciência.
Cruz, O., Raposo, J. V., Ducharne, M. A., Almeida, L. S., Teixeira, C. M., \& Fernandes, H. M. (2011). Questionário de Estilos Educativos Parentais (QEEP): Contributos para a validação factorial da versão Portuguesa das Parenting Scales. RIDEP, 1(31), 157-176.

Davis-Kean, P. (2005). The influence of parent education and family income on child achievement: The indirect role of parental expectations and the home environment. Journal of Family Psychology, 19(2), 294-304. http://dx.doi.org/10.1037/08933200.19.2.294

De Graaf, N. D., De Graaf, P. M., \& Kraaykamp, G. (2000). Parental cultural capital and educational attainment in the Netherlands: A refinement of the cultural capital perspective. Sociology of Education, 73(2), 92-111. http://dx.doi.org/10.2307/2673239

Deary, I., Strand, S., Smith, P., \& Fernandes, C. (2007). Intelligence and educational achievement. Intelligence, 35(1), 13-21. http:// dx.doi.org/10.1016/j.intell.2006.02.001

Deary, I., Taylor, M., Hart, C., Wilson, V., Smith, G., Blane, D., \& Starr. J. (2005). Intergenerational social mobility and midlife status attainment: Influences of childhood intelligence, childhood social factors, and education. Intelligence, 33(5), 455-472. http://dx.doi.org/10.1016/j.intell.2005.06.003

Dumka, L., Gonzales, N., Wheeler, L., \& Millsap, R. (2010). Parenting self-efficacy and parenting pratices over time in Mexicam American families. Journal of Family Psychology, 24(5), 522-531. http://dx.doi.org/10.1037/a0020833

Engin-Demir, C. (2009). Factors influencing the academic achievement of the Turkish urban poor. International Journal of Educational Development, 29(1), 17-29. http://dx.doi. org/10.1016/j.ijedudev.2008.03.003

Freijo, E., Oliva, A., Olabarrieta, F., Martín, J., Manzano, A., \& Richards, M. (2008). Quality of family context or sibling status? Influences on cognitive development. Early Child Development and Care, 178(2), 153-164. http://dx.doi. org/10.1080/03004430600685373

Freitas, S., Simões, M. R., Alves, L., \& Santana, I. (2012). Montreal cognitive assessment: Influence of sociodemographic and health Variables. Archives of Neuropsychology, 27(2), 165-175. http://dx.doi.org/10.1093/arclin/acr116

Galindo, C., \& Sheldon, S. (2012). School and home connections and children's kindergarten achievement: The mediating effects of family involvement. Early Childhood Research Quarterly, 27(1), 90-103. http://dx.doi.org/10.1016/j.ecresq.2011.05.004

García, J., \& Sánchez, J. M. (2005). Práticas educativas familiares y autoestima. Psicothema, 17(1), 76-82. ISSN: 0214-9915

Garcia-Bacete, F. (2003). Las relaciones escuela-familia: Un reto educativo. Infancia y aprendizaje, 26(4), 425-437. http://dx.doi. org/10.1174/021037003322553824

González, L. L. (2011). El contexto familiar como componente optimizador en el rendimento académico. Revista GalegoPortuguesa de Psicoloxía e Educación, 19(2), 147-167. ISSN: 1138-1663

Gottfredson, L. S. (2002). G: Highly general and highly practical. In R. J. Sternberg \& E. L. Grigorenko (Eds.), The general factor of intelligence: How general is it? (pp. 331-380). New Jersey: LEA.

Guo, G., \& Harris, K. M. (2000). The mechanisms mediating the effects of poverty on children's intellectual development. Demography, 37(4), 431-447. http://dx.doi.org/10.1353/ dem.2000.0005 
Gutman, L. M., Sameroff, A. J., \& Cole, R. (2003). Academic growth curve trajectories from the 1 st grade to 12 st grade: Effects of multiple social risk factors and preschool child factors. Development Psychology, 39(4), 777-790. http://dx.doi. org/10.1037/0012-1649.39.4.777

Guttman, L., \& McLoyd, V. (2000). Parents' management of their children's education within the home, at school, and in the community: An examination of african-american families living in poverty. Urban Review, 32(1), 1-24. http://dx.doi. org/10.1023/A:1005112300726

Harris, J. R. (1995). Where is the child's environment? A group socialization theory of development. Psychological Review, 102(3), 458-489. http://dx.doi.org/10.1037/0033295X.102.3.458

Hoover-Dempsey, K. V., \& Sandler, H. M. (1995). Parental involvement in children's education: Why does it make a difference. Teachers College Record, 2(97), 311-331. ISSN0161-4681

Jadue, G. (2002). Fatores psicológicos que predisponen al bajo rendimento al fracasso y a la deserción escolar afectan el rendimento escolar. Estudios Pedagógicos, 28, 193-204. http:// dx.doi.org/10.4067/S0718-07052002000100012

Jaeger, M. M. (2009). Equal access but unequal outcomes: Cultural capital and educational choice in a meritocratic society. Social Forces, 87(4), 1943-1971. https://doi.org/10.1353/sof.0.0192

Jeynes, W. (2005). A meta-analysis of the relation of parental involvement to urban elementary school student academic achievement. Urban Education, 40(3), 237-269. http://dx.doi. org/10.1177/0042085905274540

Jimerson, S., Egeland, B, Sroufe, L. A., \& Carlson, B. (2000). A prospective longitudinal study of high school dropouts examining multiple across development. Journal of School Psychology, 38(6), 525-549. http://dx.doi.org/10.1016/S00224405(00)00051-0

Johnson, W. (2010). Understanding the genetics of intelligence: Can height help? Can corn oil? Current Directions in Psychological Science, 19(3), 177-182. http://dx.doi. org/10.1177/0963721410370136

Kane, H. D., \& Brand, C. R. (2006). The variable importance of general intelligence $(\mathrm{g})$ in the cognitive ability of children and adolescents. Educational Psychology, 26(6), 751-767. http:// dx.doi.org/10.1080/01443410500390897

Lareau, A., \& Horvat, E. M. (1999). Moments of social inclusion and exclusion. Race, class, and cultural capital on family-school relationships. Sociology of Education, 72(1), 37-53. http:// dx.doi.org/10.2307/2673185

Lemos, G. C. (2007). Habilidades cognitivas e rendimento escolar entre o 5. ${ }^{\circ}$ e 12. ${ }^{\circ}$ anos de escolaridade (Tese de Doutoramento). Braga: Universidade do Minho.

Lemos, G. C., Almeida, L. S., Guisande, M. A., \& Primi, R. (2008). Inteligência e rendimento escolar: Análise da sua relação ao longo da escolaridade. Revista Portuguesa de Educação, 21, 83-99. ISSN 0871-9187

Lemos, G. C., Almeida, L., \& Colom, R. (2011). Intelligence of adolescents is related to their parents' educational level but not to family income. Personality and Individual Differences, 50(7), 1062-1067. http://dx.doi.org/10.1016/j.paid.2011.01.025
Lordelo, E. R., Chalhub, A. A., Guirra, R. C., \& Carvalho, C. S. (2007). Contexto e desenvolvimento cognitivo: Frequência à creche e evolução do desenvolvimento mental. Psicologia: Reflexão e Crítica, 20(2), 324-334. http://dx.doi.org/10.1590/ S0102-79722007000200019

Lordelo, E. R., Fonseca, A. L., \& Araújo, M. L. V. B. (2000). Responsividade do ambiente de desenvolvimento: Crenças e práticas como sistema cultural de criação de filhos. Psicologia: Reflexão e Crítica, 13(1), 73-80. http://dx.doi.org/10.1590/ S0102-79722000000100009

Mercy, J. A., \& Steelman, L. C. (1982). Familial influence on the intellectual attainment of children. American Sociological Review, 47(4), 532-542.

Myrberg, E., \& Rosén, M. (2009). Direct and indirect effects of parents' education on reading achievement among third graders in Sweden. British Journal of Educational Psychology, 79(4), 695-711. http://dx.doi.org/10.1348/000709909X453031

Nisbett, R. E., Aronson, J., Blair, C., Dickens, W., Flynn, J., Halpern, D. F., \& Turkheimer, E. (2012). Intelligence: New findings and theoretical developments. American Psychologist, 67(2), 130-159. http://dx.doi.org/10.1037/a0026699

O’Connor, E., \& McCartney, K. (2007). Examining teacher-child relationships and achievement as part of an ecological model of development. American Educational Research Journal, 44(2), 340-369. http://dx.doi.org/10.3102/0002831207302172

Phillipson, S. N. (2009). Role of parents in children's academic achievement: A specific sociocultural context. Koln, Germany: LAP LAMBERT Academic Publishing.

Phillipson, S. N. (2010). Modeling parental role in academic achievement: Comparing high-ability to low- and averageability students. Talent Development \& Excellence, 2, 83-103. ISSN: 1869-2885

Pomerantz, E. M., \& Dong, W. (2006). Effects of mothers' perceptions of children's competence: The moderating role of mother's theories of competence. Developmental Psychology, 42(5), 950-961. http://dx.doi.org/10.1037/0012-1649.42.5.950

Ribas, R. C., Jr., Moura, M. L. S., \& Bornstein, M. (2003). Socioeconomic status in Brazilian psychological research: II Socioeconomic status and parenting knowledge. Estudos de Psicologia (Natal), 8(3), 385-392 http://dx.doi.org/10.1590/ S1413-294X2003000300005

Rimkute, L., Hirvonen, R., Tolvanen, A., Aunola, K., Nurmi, J.-E. (2012). Parents' role in adolescents' educational expectations. Scandinavian Journal of Educational Research, 56(6), 571590. http://dx.doi.org/10.1080/00313831.2011.621133

Rindermann, H. (2007). The big G-factor of national cognitive ability. European Journal of Personality, 21(5), 767-787. http:// dx.doi.org/10.1002/per.658

Rindermann, H., Michou, C. D., \& Thompson, J. (2011). Children's writing ability: Effects of parent's education, mental speed and intelligence. Learning and Individual Differences, 21(5), 562568. http://dx.doi.org/10.1016/j.lindif.2011.07.010

Roazzi, A., \& Souza, B. C. (2002). Repensando a inteligência. Paidéia, 12(23), 31-55. http://dx.doi.org/10.1590/S0103863 X2002000200004

Roscigno, V. J., \& Ainsworth-Darnell, J. W. (1999). Race, cultural capital, and educational resources: Persistent inequalities and achievement returns. Sociology of Education, 72(3), 158-178. http://dx.doi.org/ 10.2307/2673227 
Rothon, C., Arephin, M., Klineberg, E., Cattell, V., \& Stansfeld, S. (2011). Structural and socio-psychological influences on adolescents' educational aspirations and subsequent academic achievement. Social Psychology of Education, 14(2), 209-231. http://dx.doi.org/10.1007/s11218-010-9140-0

Santos, P. L., \& Graminha, S. S. V. (2005). Estudo comparativo das características do ambiente familiar de crianças com alto e baixo rendimento acadêmico. Paidéia, 15(31), 217-226. http:// dx.doi.org/10.1590/S0103-863X2005000200009

Scarr, S., \& Weinberg, R. A. (1978). The influence of "family background" on intellectual attainment. American Sociological Review, 43(5), 674-692.

Seabra-Santos, M. J. (2000). Avaliação psicológica em idade préescolar: O caso da avaliação da inteligência. Psychologica, $25,143-162$

Sellers, A. H., Burns, W. J., \& Guyrke, J. (1996). Prediction of premorbid intellectual functioning of young children using demographic information. Applied Neuropsychology, 3(1), 21-27. http://dx.doi.org/10.1207/s15324826an0301_4

Spinath, B., Spinath, F. M., Harlaar, N., \& Plomin, R. (2006). Predicting school achievement from general cognitive ability, self-perceived ability and intrinsic value. Intelligence, 34(4), 363-374. http://dx.doi.org/10.1016/j.intell.2005.11.004

Stelzl, I., Merz, F., Ehlers, T., \& Remer, H. (1995). The effect of schooling of the development of fluid and crystallized intelligence: A quasi-experimental study. Intelligence, 21(3), 279-296. http://dx.doi.org/10.1016/0160-2896(95)90018-7

Sternberg, R. J. (2012). Intelligence. Wiley Interdisciplinary Reviews-cognitive Science, 3(5), 501-511. http://dx.doi. org/10.1002/wcs.1193

Strenze, T. (2007). Intelligence and socioeconomic success: A meta-analytic review of longitudinal research. Intelligence, 35(5), 401-426. http://dx.doi.org/ 10.1016/j.intell.2006.09.004

Stull, J. C. (2013). Family socioeconomic status, parent expectations, and a child's achievement. Research in Education, 90(1), 5367. http://dx.doi.org/10.7227/RIE.90.1.4
Sullivan, A. (2001). Cultural capital and educational attainment. Sociology, 35(4), 893-912. http://dx.doi.org/ $10.1177 / 0038038501035004006$

Talib, J., Mohamad, Z., \& Mamat, M. (2011). Effects of parenting style on children development. World Journal of Social Sciences, 1(2), 14-35.

Te Nijenhuis, J., Evers, A., \& Mur, J. (2000). Validity of the Differential Aptitude Test for the assessment of immigrant children. Educational Psychology, 20(1), 99-115. http://dx.doi. org/10.1080/014434100110416

Tudge, J. R., Mokrova, I., Hatfield, B. E., \& Karnik, R. (2009). Uses and misuses of Bronfenbrenner's bioecological theory of human development. Journal of Family Theory \& Review, 1(4), 198-210. http://dx.doi.org/10.1111/j.1756-2589.2009.00026.x

Umek, L. M., Podlesek, A., \& Fekonja, U. (2005). Assessing the home literacy environment: Relationships to child language comprehension and expression. European Journal of Psychological Assessment, 21(4), 271-281. http://dx.doi. org/10.1027/1015-5759.21.4.271

Vandell, D. L. (2000). Parents, peer groups, and other socializing influences. Developmental Psychology, 36(6), 699-710. http:// dx.doi.org/10.1037/0012-1649.36.6.699

Vanderploeg, R. D., Schinka, J. A., Baum, K. M., Tremont, G., \& Mittenberg, W. (1998). WISC-III premorbid prediction strategies: Demographic and best performance approaches. Psychological Assessment, 10(3), 277-284. http://dx.doi. org/10.1037/1040-3590.10.3.277

Veiga, F., Galvão, D., Festas, I., \& Taveira, C. (2012). Envolvimento dos alunos na escola: Relações com variáveis contextuais e pessoais - Uma revisão da literatura. Psicologia, Educação e Cultura, XVI(2), 36-50.

Wechsler, D. (2003). Escala de Inteligência de Wechsler para Crianças-III: Manual. Lisboa: CEGOC.

Zhan, M. (2006). Assets, parental expectations and involvement, and children's educational performance. Children and Youth Services Review, 28(8), 961-975. http://dx.doi.org/10.1016/j. childyouth.2005.10.008

Recebido em 17.12.2013

Primeira decisão editorial em 17.03.2015

Versão final em 26.03.2015

Aceito em 11.06.2015 